Article

\title{
Dynamics of an Ongoing Wolbachia Spread in the European Cherry Fruit Fly, Rhagoletis cerasi (Diptera: Tephritidae)
}

\author{
Martin Schebeck ${ }^{1}\left(\mathbb{D}\right.$, Lukas Feldkirchner ${ }^{1}$, Christian Stauffer ${ }^{1}\left[\right.$ and Hannes Schuler ${ }^{2, *} \mathbb{C}$ \\ 1 Department of Forest and Soil Sciences, University of Natural Resources and Life Sciences Vienna, BOKU, \\ Peter-Jordan-Straße 82/I, A-1190 Vienna, Austria; martin.schebeck@boku.ac.at (M.S.); \\ lukas.feldkirchner@gmail.com (L.F.); christian.stauffer@boku.ac.at (C.S.) \\ 2 Faculty of Science and Technology, Free University of Bozen-Bolzano, Universitätsplatz 5, \\ I-39100 Bozen-Bolzano, Italy \\ * Correspondence: hannes.schuler@unibz.it; Tel.: +39-0471-017648
}

Received: 16 May 2019; Accepted: 11 June 2019; Published: 14 June 2019

\begin{abstract}
Numerous terrestrial arthropods are infected with the alphaproteobacterium Wolbachia. This endosymbiont is usually transmitted vertically from infected females to their offspring and can alter the reproduction of hosts through various manipulations, like cytoplasmic incompatibility (CI), enhancing its spread in new host populations. Studies on the spatial and temporal dynamics of Wolbachia under natural conditions are scarce. Here, we analyzed Wolbachia infection frequencies in populations of the European cherry fruit fly, Rhagoletis cerasi (L.), in central Germany-an area of an ongoing spread of the CI-inducing strain $w$ Cer2. In total, 295 individuals from 19 populations were PCR-screened for the presence of $w$ Cer2 and their mitochondrial haplotype. Results were compared with historic data to understand the infection dynamics of the ongoing $w$ Cer2 invasion. An overall $w$ Cer2 infection frequency of about $30 \%$ was found, ranging from $0 \%$ to $100 \%$ per population. In contrast to an expected smooth transition from $w$ Cer2-infected to completely $w$ Cer2-uninfected populations, a relatively scattered infection pattern across geography was observed. Moreover, a strong Wolbachia-haplotype association was detected, with only a few rare misassociations. Our results show a complex dynamic of an ongoing Wolbachia spread in natural field populations of R. cerasi.
\end{abstract}

Keywords: endosymbiont; cytoplasmic incompatibility; bacterial spread; horizontal transfer; maternal transmission; reproductive manipulation; agricultural pest

\section{Introduction}

Heritable bacterial endosymbionts are present in a broad range of arthropods with intriguing effects on the ecology and evolution of numerous species [1,2]. Endosymbionts are usually transmitted vertically from infected females to their offspring. By manipulating the reproduction through the induction of cytoplasmic incompatibility (CI), parthenogenesis, feminization, and male-killing, bacterial endosymbionts increase the fitness of infected females and enhance their frequency in host populations [3,4]. Certain endosymbionts are also able to provide fitness benefits protecting their host against RNA viruses or natural enemies $[5,6]$. Most endosymbionts are able to invade and adapt to new species by interspecific horizontal transmission [7-10].

One of the most common bacterial endosymbionts that can manipulate arthropod reproduction is Wolbachia [11]. This bacterium has been estimated to infect more than $50 \%$ of terrestrial arthropod species [1] and is present in numerous hexapods, crustaceans, chelicerates, and nematodes [11]. The most common reproductive manipulation is CI that is expressed when a Wolbachia-infected male 
mates with a Wolbachia-uninfected female, or with a female harboring a different Wolbachia strain, resulting in embryonic death of fertilized eggs [12].

A Wolbachia infection can influence the mitochondrial genetic structure of the host [13]. When a Wolbachia strain spreads into a population of uninfected individuals, the mitochondrial haplotype associated with the infected individual will hitchhike with the spreading endosymbiont [13-17]. As a result, an endosymbiont infection can affect the genetic population structure of arthropod species by reducing the diversity of mitochondrial haplotypes when infected individuals-associated with a specific mitochondrial haplotype - eliminate haplotypes associated with uninfected individuals [13].

The European cherry fruit fly, Rhagoletis cerasi (L., 1758) (Diptera: Tephritidae), is a widespread insect with a complex life history. In addition to a broad spectrum of evolutionary research questions [9,16,18-20], this tephritid is also of particular interest in applied fields [21]. It infests hosts of two different plant families, that is, Prunus spp. (Rosaceae) and Lonicera spp. (Caprifoliaceae) $[18,20]$ and is therefore of significant human interest, as it is a severe pest of sweet and sour cherries [22,23].

Rhagoletis cerasi has a univoltine life cycle. In spring, prior to the ripening of fruits, adult flies emerge from their overwintering sites next to their natal host and usually do not disperse over long distances [21]. After mating on or close to host plants, usually one egg is deposited into a ripening fruit. These fruits are marked with specific pheromones to avoid an additional oviposition [24-26]. After embryonic development, larvae feed on fruits of their natal host plant. Last-instar larvae leave the fruits, dig into the soil, pupate, and overwinter in a diapausing state, completing their univoltine life cycle [21,27].

Moreover, $R$. cerasi has an intriguing reproductive biology. Crossing studies among European populations revealed strong incompatibility patterns from up to $98 \%$ when males from Southern and Central Europe mated with females from other European regions [18,19]. After the detection of Rickettsia-like organisms (RLOs) in reproductive organs [28], Riegler \& Stauffer [29] described the presence of Wolbachia in this tephritid. While all individuals are infected by the same Wolbachia strain $w$ Cer1, only populations from Southern and Central Europe are additionally infected by a second strain, $w$ Cer2 $[16,29]$. The distribution of Wolbachia strains matches the incompatibility patterns reported by Boller et al. [19], which suggests that $w$ Cer2 is causing this reproductive alteration [29].

Between southern and northern $R$. cerasi populations, there is a shift from completely $w$ Cer2-infected populations to populations not infected with $w$ Cer2 [29]. In these transitional zones, $w$ Cer2-infected and $w$ Cer2-uninfected flies coexist with gradients in infection frequencies (Figure 1a) [16,29,30]. Empirical studies combined with mathematic modelling showed that $w$ Cer 2 is currently invading $w$ Cer2-uninfected R. cerasi populations [16,30]. A recent study of two R. cerasi transects in the Czech Republic and Hungary showed a smooth gradient from completely $w$ Cer2-infected populations in the south to uninfected populations in the north and east, documenting a spatial spread of 1-2 km per generation [30].

Sequencing a part of the mitochondrial COI gene showed that $R$. cerasi exhibits a low genetic diversity in its European range, with only two haplotypes [16]. Comparison of haplotype of the fly and its Wolbachia infection status revealed a strong association between the endosymbiont and the mitochondrial haplotype-individuals of haplotype 1 (HT1) are wCer2-uninfected, whereas wCer2-infected flies are associated with haplotype 2 (HT2). Occasional misassociations have been reported from transitional zones as a result of intraspecific horizontal transfer and imperfect vertical transmission, highlighting the complex spread of this endosymbiont [16].

The invasion dynamics of Wolbachia in natural field populations are rarely studied [16,30-33]. To get detailed insights into the temporal and spatial spread of Wolbachia in R. cerasi, we performed a fine-scale sampling of 19 populations in Central Germany, where $w$ Cer2 is currently spreading. By studying Wolbachia infection frequencies, assessing mitochondrial genotypes of the host, and comparing these results with historic data [16], we aim to get new insights into the dynamics of an ongoing Wolbachia spread under natural conditions. 


\section{Materials and Methods}

\subsection{Sampling and DNA Extraction}

Samples were collected from infested fruits of Prunus avium and Lonicera xylosteum in July 2016. In total, specimens from 19 German locations were sampled: One site from the northern part of Baden-Württemberg, i.e., 16/1, and 18 populations from central-south Hesse, i.e., 16/2-16/19 (Table 1, Figure 1, Table S1). Individuals were collected from a single plant per location, either as larvae from infested fruits in the field or as pupae after emerging from cherries in the laboratory. To avoid the analysis of siblings, fruits from different parts of one tree/shrub were taken. Samples were stored in absolute ethanol at $-20{ }^{\circ} \mathrm{C}$. DNA was extracted from 16 individuals per location, except for the populations 16/5 ( $n=15), 16 / 6(n=10)$, and 16/19 ( $n=14$; Table S1), using the GenElute Mammalian Genomic DNA miniprep kit (Sigma-Aldrich, St. Louis, MO, USA) following the manufacturer's instructions.

\subsection{Wolbachia Screening}

Samples were PCR-screened for the presence of wCer1 and wCer2 using strain-specific primers targeting a part of the Wolbachia surface protein (wsp) [29,34,35]. PCR reactions were performed in a total volume of $10 \mu \mathrm{L}$, containing $1 \mathrm{mg} / \mathrm{mL}$ BSA, $2 \mathrm{mM}$ Y-buffer (PeqLab/VWR, Erlangen, Germany), $800 \mu \mathrm{M} d N T P s, 0.2 \mu \mathrm{M}$ forward and reverse primer each, $0.5 \mathrm{U}$ Taq polymerase (PeqLab/VWR), and $1 \mu \mathrm{L}$ of template DNA. PCR conditions were $2 \mathrm{~min}$ at $94{ }^{\circ} \mathrm{C}$, followed by 35 cycles of $94{ }^{\circ} \mathrm{C}$ for $30 \mathrm{~s}$, $55{ }^{\circ} \mathrm{C}$ for $45 \mathrm{~s}, 72{ }^{\circ} \mathrm{C}$ for $1 \mathrm{~min}$, followed by a final extension at $72{ }^{\circ} \mathrm{C}$ for $10 \mathrm{~min}$.

PCR-amplified fragments were electrophoretically separated on a $2 \%$ agarose gel stained with GelRed Nucleic Acid Dye (Biotum, Hayward, CA, USA). As wCer1 is fixed in European R. cerasi $[16,29,30,35]$, screenings for this strain were performed to control for sufficient DNA quality. In order to avoid false-negatives, all results were confirmed by two independent PCR runs.

\subsection{Mitochondrial Genotyping of $R$. cerasi}

To study the association of $R$. cerasi mitochondrial haplotypes with the Wolbachia infection status, genotyping of the flies was performed by applying restriction fragment length polymorphism (RFLP) as used in [16]. In brief, a part of the mitochondrial COI gene was PCR-amplified using the primers Pat and Dick [36]. Following PCR (conditions were the same as described above), $10 \mu \mathrm{L}$ of the PCR product was incubated with $2 \mathrm{U}$ of HaeIII (Thermo Fisher Scientific, Waltham, MA, USA) at $37^{\circ} \mathrm{C}$ for $3 \mathrm{~h}$. Fragments were separated on a $\%$ agarose gel-HT2 is cut into a $342 \mathrm{bp}$ and a 204 bp fragment, while HT1 remains undigested [16].

\subsection{Comparison of Our Results with Historic Data}

Eight out of the $19 R$. cerasi populations studied here were already Wolbachia-screened and genotyped between 1999 and 2014 [16]: Dossenheim (16/1), Stockstadt (16/3), Ober-Ramstadt (16/4), Lich (16/12), Gießen (16/13, 16/14), Lahnau (16/15), and Alsfeld (16/16). To assess the spatial and temporal dynamics of $w$ Cer2, infection frequencies of this strain of the various years were compared.

\section{Results}

\subsection{Wolbachia Infection Frequencies}

Screening of $R$. cerasi for the presence of wCer2 revealed that $30.2 \%$ of samples (89 out of 295) were infected with this strain (Table 1, Figure 1c, Table S1). In three out of 19 locations, wCer2 was fixed: In the southernmost site Dossenheim (16/1), and in two central locations Hailer (16/8) and Gießen/Prunus (16/14). In contrast, the three populations Rosbach (16/7), Lich (16/12), and Wallenrod $(16 / 17)$ were completely wCer2-uninfected. wCer2 infection frequencies in the other 13 sites ranged from 6.3\% in Bensheim (16/2) and Ober-Ramstadt (16/4) to 75.0\% in Gießen/Lonicera (16/13) (Table 1, Figure 1c, Table S1). 
Table 1. Infection frequencies of $w$ Cer2 across different $R$. cerasi populations and ratios of wCer2-infected flies associated with haplotype 2 (HT2) per population ${ }^{*}=$ populations with Wolbachia-haplotype misassociations).

\begin{tabular}{cccc}
\hline Population \# & Location & \% wCer2 & \% wCer2/HT2 \\
\hline $16 / 1$ & Dossenheim & 100 & 100 \\
$16 / 2$ & Bensheim & 6.3 & 6.3 \\
$16 / 3$ & Stockstadt & 18.8 & 18.8 \\
$16 / 4$ & Ober-Ramstadt & 6.3 & 6.3 \\
$16 / 5$ & Erbenheim & 6.7 & 6.7 \\
$16 / 6$ & Idstein & 10.0 & 10.0 \\
$16 / 7$ & Rosbach & 0.0 & 0.0 \\
$16 / 8$ & Hailer & 100 & 100 \\
$16 / 9$ & Weckesheim & 25.0 & 25.0 \\
$16 / 10$ & Utphe & 12.5 & 12.5 \\
$16 / 11$ & Langsdorf & 18.8 & 18.8 \\
$16 / 12$ & Lich & 0.0 & 0.0 \\
$16 / 13$ & Gießen/Lonicera & 75.0 & $62.5 *$ \\
$16 / 14$ & Gießen/Prunus & 100 & $68.8 *$ \\
$16 / 15$ & Lahnau & 18.8 & $0.0 *$ \\
$16 / 16$ & Alsfeld & 12.5 & 12.5 \\
$16 / 17$ & Wallenrod & 0.0 & 0.0 \\
$16 / 18$ & Schlüchtern & 37.5 & 37.5 \\
$16 / 19$ & Grossenmoor & 14.3 & 14.3 \\
\hline
\end{tabular}

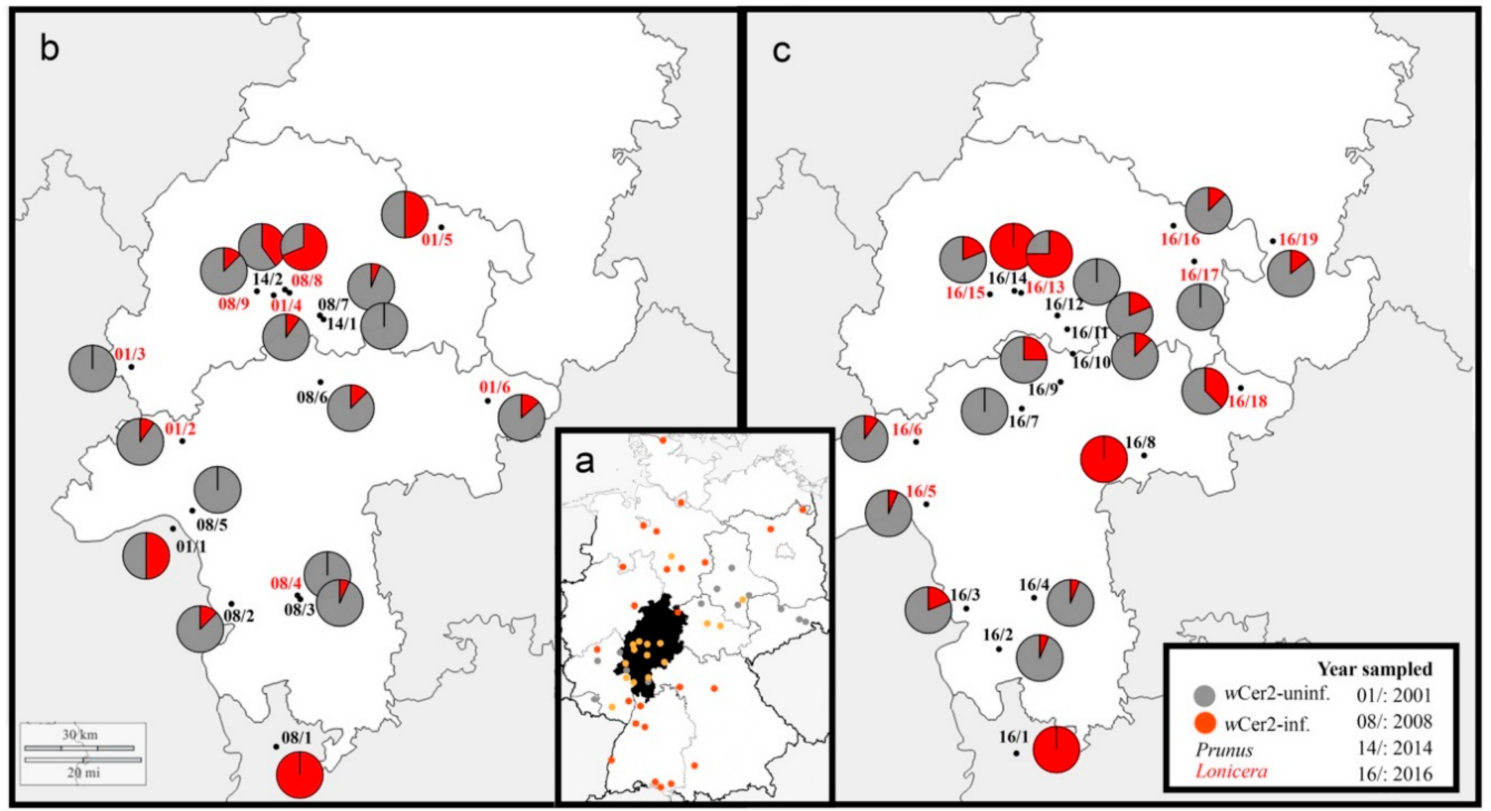

Figure 1. Geographic distribution of Wolbachia of R. cerasi: (a) Schematic overview of Wolbachia infections across Germany. Distribution of wCer2-uninfected (grey dots), wCer2-infected (red dots), and transitional populations with $w$ Cer2-infected and $w$ Cer2-uninfected flies (orange dots) sampled between 2000-2008, modified from [16]. Black-filled state represents Hesse, the central study site; (b) $w$ Cer2 infection frequencies between 2001 and 2014 and (c) in 2016. Grey = proportion of $w$ Cer2-uninfected flies, red $=$ proportion of $w$ Cer2- infected flies, black numbers represent flies collected from Prunus and red numbers represent flies collected from Lonicera.

$w$ Cer2 frequencies of the $R$. cerasi populations studied here exhibit no distinct transitional infection pattern with smooth gradients from completely wCer2-infected to entirely wCer2-uninfected populations [30] (Figure 1c). The southernmost site Dossenheim (16/1) was completely wCer2-infected, whereas the population in Bensheim (16/2), less than $30 \mathrm{~km}$ further north, showed a low infection 
frequency of $6.3 \%$. wCer2 frequencies in the adjacent locations Stockstadt (16/3) and Ober-Ramstadt (16/4), $14 \mathrm{~km}$ north-west and $17 \mathrm{~km}$ further north-east, respectively, ranged from $6.3 \%$ to $18.8 \%$ (Figure 1c). The wCer2 infection frequency was similar in Erbenheim (16/5) and Idstein (16/6), $30 \mathrm{~km}$ and $50 \mathrm{~km}$ further north, with $6.7 \%$ and $10 \%$, respectively. The population in Rosbach (16/7), however, was already completely $w$ Cer2-uninfected. In contrast, $25 \%$ of the individuals from the population in Weckesheim (16/9), $13 \mathrm{~km}$ further north-west, were wCer2-infected, whereas all individuals from Hailer (16/8), $30 \mathrm{~km}$ further south-east, were infected by wCer2 (Figure 1c).

A similar pattern was found in the northern locations where a wCer2-uninfected population (Lich, 16/12) was surrounded by two transitional populations 5 and $12 \mathrm{~km}$ apart, and just $14 \mathrm{~km}$ distant from the completely wCer2-infected population in Gießen/Prunus (16/14) (Figure 1c).

\subsection{Mitochondrial Genotyping of R. cerasi and Haplotype-Wolbachia Associations}

As only two mitochondrial R. cerasi haplotypes across Europe have been described previously, we used an RFLP approach to determine the haplotype affiliation of each individual. Genotyping of flies showed that the Wolbachia infection status was strongly associated with the two mitochondrial haplotypes of the host (Figure $2 \mathrm{~b}$ ). Almost all wCer2-uninfected individuals were associated with HT1 (205 out of 206). Just one individual from Alsfeld (16/16) was wCer2-uninfected but associated with HT2. In contrast, $88.8 \%$ of $w$ Cer2-infected $R$. cerasi were associated with HT2, whereas 10 wCer2-infected individuals from three populations were associated with HT1 (Figure 2b).

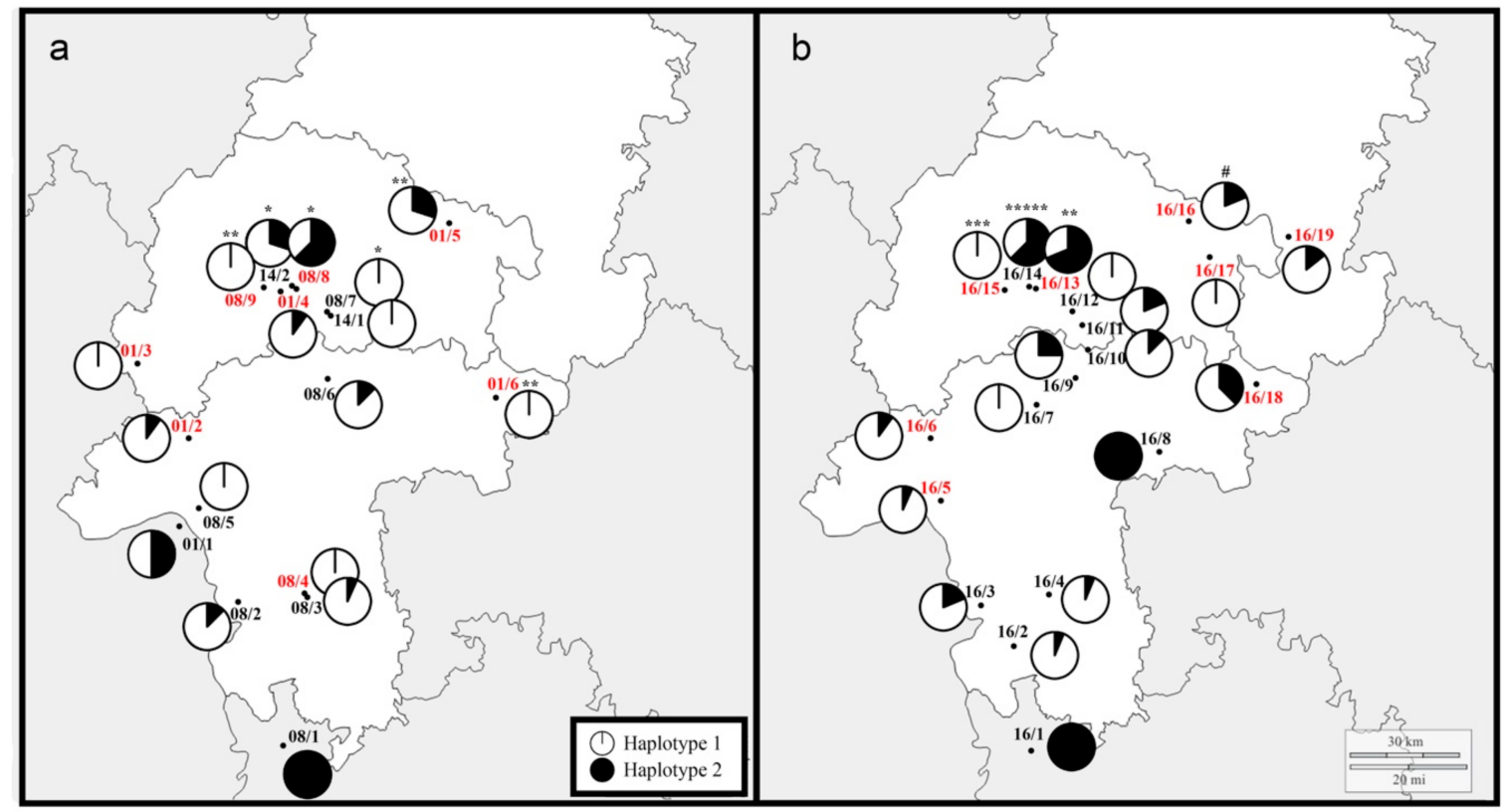

Figure 2. (a) Prevalence of haplotypes between 2001 and 2014 and (b) in 2016. White = proportion of individuals associated with HT1, black = proportion of individuals with HT2. Asterisks represent number of $w$ Cer2-infected individuals associated with HT1, whereas the pound represents the only individual that was wCer2-uninfected but associated with HT2.

Haplotype-Wolbachia misassociations were found in populations in geographically close proximity, i.e., Gießen/Lonicera (16/13), Gießen/Prunus (16/14), and Lahnau (16/15). In Gießen/Lonicera (16/13), two of the 12 wCer2-infected samples were associated with HT1, whereas in Lahnau all three wCer2-infected flies were associated with HT1. The most significant deviation was found in Gießen/Prunus (16/14), where all 16 individuals were wCer2-infected, but only 11 (68.8\%) were associated with HT2. 


\subsection{Wolbachia Dynamics and Haplotype Associations in Time and Space}

Out of the 19 R. cerasi populations studied here, eight had already been screened for their Wolbachia infection status and mitochondrial haplotypes between 1999 and 2014 [16]. We compared our results with these historic data to infer the dynamics of Wolbachia infection and the associated mitochondrial haplotype of the fly. Rhagoletis cerasi collected from Dossenheim (16/1)—where all individuals screened were infected by wCer2-was already completely invaded by this strain in 1999 and 2008 (Table 2). In Stockstadt (16/3) and Lahnau (16/15), wCer2 infection frequencies increased from $12.5 \%$ in 2008 to $18.8 \%$ in 2016 in both locations, however, wCer2 frequencies did not differ significantly among these years (both locations: $\left.\chi^{2}=0.237, p=0.434\right)$. In Ober-Ramstadt $(16 / 4)$, the ratio of infected individuals remained constant-in 2008, one out of 15 flies (6.7\%), and in 2016, one out of 16 flies (6.3\%) was $w$ Cer2-infected $\left(\chi^{2}=0.002, p=0.499\right)$. In 2008 , the population in Lich $(16 / 12)$ had a $w$ Cer2 infection frequency of $6.3 \%$, however, none of the flies were $w$ Cer2-infected in $2014\left(\chi^{2}=0.650, p=0.339\right)$ and in $2016\left(\chi^{2}=1.032, p=0.297\right)$. A significant decrease of $w$ Cer2 occurred in the $R$. cerasi population of Alsfeld (16/16), where 50\% of individuals were $w$ Cer2-infected in 2000, while in 2016, just $12.5 \%$ of the samples were infected by this strain $\left(\chi^{2}=4.398, p=0.036\right)$. Moreover, we found a potential effect of the host plant of the fly on its Wolbachia infection. In Gießen (16/13 and 16/14), flies collected from Lonicera had a significant increase of wCer2 from $10 \%$ in 2001 to $68.8 \%$ in $2014\left(\chi^{2}=8.547, p=0.003\right)$. Comparing infection frequencies from the same site (16/13) in 2016 revealed a further increase in infection frequencies to $75 \%\left(\chi^{2}=0.155, p=0.456\right)$. Rhagoletis cerasi collected from Prunus (16/14), however, was already completely wCer2-infected.

Comparing Wolbachia-haplotype associations between different years revealed that $w$ Cer2-infected flies associated with HT1 occurred in the same populations before (Figure 2a). Overall, the number of wCer2-infected R. cerasi associated with HT1 increased in all three populations. In contrast, the population in Alsfeld (16/16), where one wCer2-uninfected individual was associated with HT2, did not show this pattern in 2001. However, two out of five wCer2-infected flies were associated with HT1 in 2001, a pattern that was not confirmed in 2016 (Table S2).

Table 2. Comparison of $w$ Cer2 infection frequencies among the years 1999 (99), 2001 (01), 2008 (08), and 2016 (16).

\begin{tabular}{cccccc}
\hline Location & Population \# & Host & Year & $n$ & \% wCer2 \\
\hline \multirow{3}{*}{ Dossenheim } & $99 / 1$ & Lonicera & 1999 & 10 & 100 \\
& $08 / 1$ & Prunus & 2008 & 16 & 100 \\
& $16 / 1$ & Prunus & 2016 & 16 & 100 \\
\hline \multirow{3}{*}{ Ober-Ramstadt } & $08 / 3$ & Lonicera & 2008 & 15 & 0 \\
& $08 / 4$ & Prunus & 2008 & 15 & 6.7 \\
& $16 / 4$ & Prunus & 2016 & 16 & 6.3 \\
\hline \multirow{2}{*}{ Stockstadt } & $08 / 2$ & Prunus & 2008 & 16 & 12.5 \\
& $16 / 3$ & Prunus & 2016 & 16 & 18.8 \\
\hline \multirow{3}{*}{ Lich } & $08 / 7$ & Prunus & 2008 & 16 & 6.3 \\
& $14 / 1$ & Prunus & 2014 & 10 & 0 \\
& $16 / 12$ & Prunus & 2016 & 16 & 0 \\
\hline \multirow{2}{*}{ Gießen } & $01 / 4$ & Lonicera & 2001 & 10 & 10 \\
& $08 / 8$ & Lonicera & 2008 & 16 & 68.8 \\
& $16 / 13$ & Lonicera & 2016 & 16 & 75 \\
& $16 / 14$ & Prunus & 2016 & 16 & 100 \\
\hline \multirow{2}{*}{ Lahnau } & $08 / 9$ & Lonicera & 2008 & 16 & 12.5 \\
& $16 / 15$ & Prunus & 2016 & 16 & 18.8 \\
\hline \multirow{2}{*}{ Alsfeld } & $01 / 5$ & Lonicera & 2001 & 10 & 50 \\
& $16 / 16$ & Lonicera & 2016 & 16 & 12.5 \\
\hline
\end{tabular}




\section{Discussion}

Here, we studied the frequency of $w$ Cer2 in R. cerasi in the central part of Germany, a region where this strain is currently spreading. Compared to previous work in this area [16], our fine-scale sampling and screening of 19 populations allowed us to characterize the spatial distribution of $w$ Cer2 in this transition zone. Furthermore, comparing our results with historic data from the last two decades [16] allowed us to assess the temporal dynamics of this Wolbachia strain in certain populations. In contrast to our expectation of a transitional zone with smooth gradients from completely infected to entirely uninfected flies, as described in other parts of the species' range [30], we found a surprisingly scattered pattern of $w$ Cer2 infection frequencies across different populations. Comparison of the Wolbachia infection status and the mitochondrial haplotype of individuals showed a strong relationship, however, occasional misassociations suggest events of intraspecific horizontal transfer. Taken together, our data provide new insights into the ongoing Wolbachia spread in European R. cerasi.

\subsection{Wolbachia Infection Frequencies in Time and Space}

At least five Wolbachia strains have been described from R. cerasi, and one individual fly can harbor various strains $[29,35]$. The spread of the strain $w$ Cer2 in Central Europe from south to north represents a rare event of an ongoing Wolbachia invasion in natural field populations $[16,29,30]$. By screening 295 individuals from 19 populations, we found an overall $w$ Cer2 infection frequency of about $30 \%$, ranging from $0 \%$ to $100 \%$ per population. Instead of gradual transitional zones between completely $w$ Cer2-infected and entirely $w$ Cer2-uninfected R. cerasi populations [30], a relatively scattered infection pattern was found. Populations completely infected with $w$ Cer2 were in close proximity to locations with low $w$ Cer2 infection frequencies. Our results, together with previous data $[16,29,30]$, provide a comprehensive picture of an ongoing Wolbachia spread in natural field populations of $R$. cerasi and suggest that the spatial pattern of this endosymbiont infection is influenced by various factors, such as long distance migration of flies and/or passive movement with infested cherries.

Although Wolbachia is one of the best-studied bacterial endosymbionts, only a limited number of studies have given insight into its spatial dynamics under natural field conditions [16,30,31,37,38]. In addition to empirical observations, theoretical modelling can help to provide a basic understanding on the mode of Wolbachia spread [14,39,40]. One of the best studied examples of an ongoing Wolbachia spread in the field is the mosquito Aedes aegypti, artificially infected with the strain $w \mathrm{Mel}$, that naturally occurs in Drosophila melanogaster [41,42]. wMel was found to spread relatively slowly in release areas with a rate of 100-200 m per year [41]. Major factors influencing this spread are deceased fitness of transinfected mosquitoes and a low dispersal rate of the host [41]. This is in contrast to the estimated spread of $w$ Cer2 in central Europe of 1-1.9 $\mathrm{km}$ per year [30], a spread that might benefit from the long adaptation of $w$ Cer2 to its host-with expected low fitness costs-and the higher dispersal capacity of the fly. Both systems showed that human-mediated dispersal of insects can influence their long-range migration and can result in unexpected spatial patterns of host organisms and their associated symbionts [16,43].

Generally, Wolbachia that causes fitness costs in its host needs to reach a sufficiently high equilibrium frequency to get established in a host population [41]. Thus, infections in low frequencies do not result in an establishment of the bacterium [40]. In contrast, Wolbachia can be established in a host population even from very low initial infection frequencies by providing positive fitness effects to their host, as it was reported from Drosophila simulans in Australia [37] and California [31]. Although the spatial pattern of $w$ Cer2 infection rates in Germany is different from other regions in the range of $R$. cerasi $[16,29,30]$, our data suggest that a certain equilibrium infection frequency in a population is necessary for this strain to become established $[16,30]$. This was shown by the comparison of Wolbachia infection frequencies at the same sites among different years. For example, in the locations Ober-Ramstadt (16/3), Stockstadt (16/4), Idstein (16/6), Lich (16/12), and Lahnau (16/15), infection frequencies remained at low levels over a period of eight to 15 years. In contrast, in Gießen/Lonicera (16/13), wCer2 infection frequencies of $R$. cerasi collected from honeysuckle increased rapidly from 
$10 \%$ in 2001 , to $68.8 \%$ in 2008 [16], and to $75 \%$ in 2016 . We assume that $w$ Cer2 reached a sufficiently high infection frequency over time, and we expect that this strain will get fixed in the following years. The importance of an equilibrium infection frequency is further supported by the absence of intermediate infection frequencies over the sampling years, as it was found in almost all locations.

In Gießen/Prunus (16/14), however, R. cerasi was already completely wCer2 infected. This suggests an influence of the fly's host plant on the Wolbachia infection dynamics. Some lines of evidence propose host plant-related differentiation patterns in R. cerasi. For example, individuals from Prunus and Lonicera show slightly different eclosion times in spring, maybe a response to a differing fruiting phenology of the host [44]. Potential ecological differentiation between host ecotypes of $R$. cerasi infesting cherry or honeysuckle $[18,20]$ might reduce gene flow between different populations, influencing the Wolbachia infection dynamics. A contrasting pattern was observed in the northernmost location Alsfeld (16/16) where the ratio of $w$ Cer2 infections decreased significantly from $50 \%$ in 2000 to $12.5 \%$ in 2016. Since none of the $w$ Cer2-uninfected individuals had the mitochondrial haplotype HT2, we exclude events of occasional loss of $w$ Cer2 as a reason for the decrease of this strain over time. The accidental anthropogenic introduction of $w$ Cer2-uninfected flies into this region, for example, via trade with R. cerasi-infested cherries, could explain this finding.

\subsection{Wolbachia Infection and Mitochondrial Haplotype of the Host}

The genetic structure of European R. cerasi on the mitochondrial level is generally low, reflected by the presence of only two haplotypes [16]. This low genetic diversity might be the result of a previous Wolbachia sweep by the strain $w$ Cer1. Schuler et al. [16] hypothesized that a few individuals of $R$. cerasi with HT1 got previously infected by $w$ Cer1 via horizontal transmission. This strain might have provided fitness benefits to the fly and swept through host populations, replacing all other haplotypes. Later, HT2 evolved, acquired wCer2 horizontally, and is now hitchhiking through European populations [16].

The mitochondrial haplotypes are tightly associated with Wolbachia, where wCer2-uninfected flies are associated with HT1 and wCer2-infected individuals with HT2. In our study, out of 295 samples, only 11 had a different Wolbachia-haplotype association. In 10 cases, these misassociations represented a wCer2-infection in individuals with HT1. This finding can be a result of intraspecific horizontal transfer [45]. For example, parasitoid wasps are known to be a potential source of horizontal transmission among species [16,45-48]. Parasitoid species of $R$. cerasi were found to be infected with Wolbachia. Their role in horizontal transmission between and within fly species, however, has yet to be studied [45]. An additional mechanism of horizontal transfer might be via cannibalism among R. cerasi larvae [45]. Although multiple larvae within one cherry are uncommon [26], under certain environmental conditions [26] or when resources for oviposition are scarce, several larvae might develop in one fruit. In this case, a larva could acquire Wolbachia by feeding on a co-occurring larva but would remain associated with its mitochondrial haplotype.

Since all cases of a wCer2-infection in HT1 flies were found exclusively in transitional populations (where flies with both Wolbachia infection types are present), this phenomenon was interpreted as transient, where HT1 is assumed to be lost in populations completely invaded by $w$ Cer2 [16]. We found just three populations in a restricted area-in Gießen (16/13 and 16/14) and Lahnau (16/15)—with individuals belonging to HT1 harboring $w$ Cer2. The comparison with historic data from 2001, 2008, and 2014 [16], however, showed a general increase of individuals with these misassociations. In the location Gießen/Prunus (16/14) where R. cerasi is completely $w$ Cer2-infected, $31 \%$ of the individuals were associated with HT1. Subsequent studies are needed to understand if these flies can form a stable co-existence of $w$ Cer2 and HT1 or if they will be lost in future generations, as simulated by Schuler et al. [16].

Finally, one single $w$ Cer2-uninfected individual associated with HT2 was found. This suggests a potential case of unsuccessful maternal transmission of Wolbachia. The low occurrence of these 
misassociations reflects a strong CI-inducing effect of $w$ Cer2 with nearly perfect transmission from females to their offspring [16,19].

\section{Conclusions}

Our screening of the European cherry fruit fly with focus on the CI-inducing strain $w$ Cer2 provides new insights into this unique endosymbiont-host system. In contrast to an expected smooth transition and continuous gradient of infection frequencies from $w$ Cer2-infected to completely uninfected R. cerasi populations [30], we found a rather scattered geographic infection pattern of $w$ Cer2 infections. Our combined analysis of the Wolbachia infection status and the associated host genotype show a highly complex picture of just partially increasing $w$ Cer2 frequencies, possibly shaped by endosymbiont losses, intraspecific horizontal transmission events, and potential anthropogenic effects.

Supplementary Materials: The following are available online at http://www.mdpi.com/2075-4450/10/6/172/s1, Table S1: Detailed overview on the Wolbachia infection status and mitochondrial haplotypes of $R$. cerasi, Table S2: Overview on historic data on the Wolbachia infection status and the mitochondrial genotypes of $R$. cerasi.

Author Contributions: Conceptualization, C.S. and H.S.; formal analysis, M.S., L.F., H.S.; writing-original draft preparation, review and editing, M.S., L.F., C.S., H.S.; visualization, H.S.; project administration, C.S., H.S.; funding acquisition, C.S., H.S.

Funding: Open Access Funding by the Austrian Science Fund (FWF). This research was funded by the Austrian Science Fund (FWF), grant numbers P26749-B25 and I2604-B25 (to C.S.) and J-3527-B22 and P31441-B29 (to H.S.).

Acknowledgments: We thank Heidrun Vogt (JKI Dossenheim, Germany) for providing samples and Susanne Krumböck (BOKU Vienna, Austria) for conducting genetic laboratory work.

Conflicts of Interest: The authors declare no conflict of interest. The funders had no role in the design of the study; in the collection, analyses, or interpretation of data; in the writing of the manuscript, or in the decision to publish the results.

\section{References}

1. Weinert, L.A.; Araujo-Jnr, E.V.; Ahmed, M.Z.; Welch, J.J. The incidence of bacterial endosymbionts in terrestrial arthropods. Proc. R. Soc. Lond. Biol. 2015, 282, 20150249. [CrossRef] [PubMed]

2. McFall-Ngai, M.; Hadfield, M.G.; Bosch, T.C.G.; Carey, H.V.; Domazet-Loso, T.; Douglas, A.E.; Dubilier, N.; Eberl, G.; Fukami, T.; Gilbert, S.F.; et al. Animals in a bacterial world, a new imperative for the life sciences. Proc. Natl. Acad. Sci. USA 2013, 110, 3229-3236. [CrossRef] [PubMed]

3. Moran, N.A.; McCutcheon, J.P.; Nakabachi, A. Genomics and evolution of heritable bacterial symbionts. Annu. Rev. Genet. 2008, 42, 165-190. [CrossRef] [PubMed]

4. Engelstadter, J.; Hurst, G.D.D. The ecology and evolution of microbes that manipulate host reproduction. Annu. Rev. Ecol. Evol. Syst. 2009, 40, 127-149. [CrossRef]

5. Teixeira, L.; Ferreira, A.; Ashburner, M. The bacterial symbiont Wolbachia induces resistance to RNA viral infections in Drosophila melanogaster. PLoS Biol. 2008, 6, 2753-2763. [CrossRef] [PubMed]

6. Jaenike, J.; Unckless, R.; Cockburn, S.N.; Boelio, L.M.; Perlman, S.J. Adaptation via symbiosis: Recent spread of a Drosophila defensive symbiont. Science 2010, 329, 212-215. [CrossRef] [PubMed]

7. O'Neill, S.L.; Giordano, R.; Colbert, A.M.E.; Karr, T.L.; Robertson, H.M. 16S ribosomal-RNA phylogenetic analysis of the bacterial endosymbionts associated with cytoplasmic incompatibility in insects. Proc. Natl. Acad. Sci. USA 1992, 89, 2699-2702. [CrossRef]

8. Caspi-Fluger, A.; Inbar, M.; Mozes-Daube, N.; Katzir, N.; Portnoy, V.; Belausov, E.; Hunter, M.S.; Zchori-Fein, E. Horizontal transmission of the insect symbiont Rickettsia is plant-mediated. Proc. R. Soc. Lond. Biol. 2012, 279, 1791-1796. [CrossRef]

9. Schuler, H.; Bertheau, C.; Egan, S.P.; Feder, J.L.; Riegler, M.; Schlick-Steiner, B.C.; Steiner, F.M.; Johannesen, J.; Kern, P.; Tuba, K.; et al. Evidence for a recent horizontal transmission and spatial spread of Wolbachia from endemic Rhagoletis cerasi (Diptera: Tephritidae) to invasive Rhagoletis cingulata in Europe. Mol. Ecol. 2013, 22, 4101-4111. [CrossRef] 
10. Gonella, E.; Pajoro, M.; Marzorati, M.; Crotti, E.; Mandrioli, M.; Pontini, M.; Bulgari, D.; Negri, I.; Sacchi, L.; Chouaia, B.; et al. Plant-mediated interspecific horizontal transmission of an intracellular symbiont in insects. Sci. Rep. 2015, 5, 15811. [CrossRef]

11. Werren, J.H.; Baldo, L.; Clark, M.E. Wolbachia: Master manipulators of invertebrate biology. Nat. Rev. Microbiol. 2008, 6, 741-751. [CrossRef] [PubMed]

12. Hoffmann, A.A.; Turelli, M. Cytoplasmic incompatibility in insects. In Influential Passengers: Inherited Microorganisms and Arthropod Reproduction; O’Neill, S., Hoffmann, A.A., Werren, J.H., Eds.; Oxford University Press: Oxford, UK, 1997; pp. 42-80.

13. Hurst, G.D.D.; Jiggins, F.M. Problems with mitochondrial DNA as a marker in population, phylogeographic and phylogenetic studies: The effects of inherited symbionts. Proc. R. Soc. Lond. Biol. 2005, 272, 1525-1534. [CrossRef] [PubMed]

14. Turelli, M.; Hoffmann, A.A.; Mckechnie, S.W. Dynamics of cytoplasmic incompatibility and mtDNA variation in natural Drosophila simulans populations. Genetics 1992, 132, 713-723. [PubMed]

15. Narita, S.; Nomura, M.; Kato, Y.; Fukatsu, T. Genetic structure of sibling butterfly species affected by Wolbachia infection sweep: Evolutionary and biogeographical implications. Mol. Ecol. 2006, 15, 1095-1108. [CrossRef] [PubMed]

16. Schuler, H.; Koppler, K.; Daxbock-Horvath, S.; Rasool, B.; Krumbock, S.; Schwarz, D.; Hoffmeister, T.S.; Schlick-Steiner, B.C.; Steiner, F.M.; Telschow, A.; et al. The hitchhiker's guide to Europe: The infection dynamics of an ongoing Wolbachia invasion and mitochondrial selective sweep in Rhagoletis cerasi. Mol. Ecol. 2016, 25, 1595-1609. [CrossRef] [PubMed]

17. Cariou, M.; Duret, L.; Charlat, S. The global impact of Wolbachia on mitochondrial diversity and evolution. J. Evolut. Biol. 2017, 30, 2204-2210. [CrossRef] [PubMed]

18. Boller, E.F.; Bush, G.L. Evidence for genetic variation in populations of European cherry fruit fly, Rhagoletis cerasi (Diptera: Tephritidae) based on physiological parameters and hybridization experiments. Entomol. Exp. Appl. 1974, 17, 279-293. [CrossRef]

19. Boller, E.F.; Russ, K.; Vallo, V.; Bush, G.L. Incompatible races of European cherry fruit fly, Rhagoletis cerasi (Diptera:Tephritidae), their origin and potential use in biological control. Entomol. Exp. Appl. 1976, 20, 237-247. [CrossRef]

20. Schwarz, D.; McPheron, B.A.; Hartl, G.B.; Boller, E.F.; Hoffmeister, T.S. A second case of genetic host races in Rhagoletis? A population genetic comparison of sympatric host populations in the European cherry fruit fly, Rhagoletis cerasi. Entomol. Exp. Appl. 2003, 108, 11-17. [CrossRef]

21. Boller, E.F.; Prokopy, R.J. Bionomics and management of Rhagoletis. Annu. Rev. Entomol. 1976, 21, $223-246$. [CrossRef]

22. Boller, E.F.; Haisch, A.; Russ, K.; Vallo, V. Economic importance of Rhagoletis cerasi L., the feasibility of genetic control and resulting research problems. Entomophaga 1970, 15, 305-313. [CrossRef]

23. Daniel, C.; Grunder, J. Integrated management of European cherry fruit fly Rhagoletis cerasi (L.): Situation in Switzerland and Europe. Insects 2012, 3, 956-988. [CrossRef] [PubMed]

24. Aluja, M.; Boller, E.F. Host marking pheromone of Rhagoletis cerasi: Field deployment of synthetic pheromone as a novel cherry fruit fly management strategy. Entomol. Exp. Appl. 1992, 65, 141-147. [CrossRef]

25. Aluja, M.; Boller, E.F. Host marking pheromone of Rhagoletis cerasi: Foraging behavior in response to synthetic pheromonal isomers. J. Chem. Ecol. 1992, 18, 1299-1311. [CrossRef] [PubMed]

26. Boller, E.F.; Aluja, M. Oviposition deterring pheromone in Rhagoletis cerasi L. Biological activity of 4 synthetic isomers and HMP discrimination of two host races as measured by an improved laboratory bioassay. J. Appl. Entomol. 1992, 113, 113-119. [CrossRef]

27. Papanastasiou, S.A.; Papadopoulos, N.T. Description of Rhagoletis cerasi (Diptera: Tephritidae) pupal developmental stages: Indications of prolonged diapause. J. Insect Sci. 2014, 14, 156. [CrossRef] [PubMed]

28. Blümel, S.; Keck, M.; Nowotny, N.; Fiedler, W.; Russ, K. Detection and therapy of Rickettsia-like-organisms (RLOs) in ovaries of the European cherry fruit fly (Rhagoletis cerasi L.; Trypetidae): An evaluation of their influence on the unidirectional crossing sterility. Pflanzenschutzberichte 1991, 52, 41-47.

29. Riegler, M.; Stauffer, C. Wolbachia infections and superinfections in cytoplasmically incompatible populations of the European cherry fruit fly Rhagoletis cerasi (Diptera, Tephritidae). Mol. Ecol. 2002, 11, 2425-2434. [CrossRef]

30. Bakovic, V.; Schebeck, M.; Telschow, A.; Stauffer, C.; Schuler, H. Spatial spread of Wolbachia in Rhagoletis cerasi populations. Biol. Lett. 2018, 14, 20180161. [CrossRef] 
31. Turelli, M.; Hoffmann, A.A. Rapid spread of an inherited incompatibility factor in California Drosophila. Nature 1991, 353, 440-442. [CrossRef]

32. Turelli, M.; Cooper, B.S.; Richardson, K.M.; Ginsberg, P.S.; Peckenpaugh, B.; Antelope, C.X.; Kim, K.J.; May, M.R.; Abrieux, A.; Wilson, D.A.; et al. Rapid global spread of $w$ Ri-like Wolbachia across multiple Drosophila. Curr. Biol. 2018, 28, 963-971. [CrossRef] [PubMed]

33. Kriesner, P.; Conner, W.R.; Weeks, A.R.; Turelli, M.; Hoffmann, A.A. Persistence of a Wolbachia infection frequency cline in Drosophila melanogaster and the possible role of reproductive dormancy. Evolution 2016, 70, 979-997. [CrossRef] [PubMed]

34. Braig, H.R.; Zhou, W.G.; Dobson, S.L.; O'Neill, S.L. Cloning and characterization of a gene encoding the major surface protein of the bacterial endosymbiont Wolbachia pipientis. J. Bacteriol. 1998, 180, 2373-2378. [PubMed]

35. Arthofer, W.; Riegler, M.; Schneider, D.; Krammer, M.; Miller, W.J.; Stauffer, C. Hidden Wolbachia diversity in field populations of the European cherry fruit fly, Rhagoletis cerasi (Diptera, Tephritidae). Mol. Ecol. 2009, 18, 3816-3830. [CrossRef] [PubMed]

36. Simon, C.; Frati, F.; Beckenbach, A.; Crespi, B.; Liu, H.; Flook, P. Evolution, weighting, and phylogenetic utility of mitochondrial gene sequences and a compilation of conserved polymerase chain reaction primers. Ann. Entomol. Soc. Am. 1994, 87, 651-701. [CrossRef]

37. Kriesner, P.; Hoffmann, A.A.; Lee, S.F.; Turelli, M.; Weeks, A.R. Rapid sequential spread of two Wolbachia variants in Drosophila simulans. PLoS Pathog. 2013, 9, e1003607. [CrossRef] [PubMed]

38. Atyame, C.M.; Labbe, P.; Rousset, F.; Beji, M.; Makoundou, P.; Duron, O.; Dumas, E.; Pasteur, N.; Bouattour, A.; Fort, P.; et al. Stable coexistence of incompatible Wolbachia along a narrow contact zone in mosquito field populations. Mol. Ecol. 2015, 24, 508-521. [CrossRef]

39. Turelli, M.; Hoffmann, A.A. Cytoplasmic incompatibility in Drosophila simulans-Dynamics and parameter estimates from natural populations. Genetics 1995, 140, 1319-1338.

40. Barton, N.H.; Turelli, M. Spatial waves of advance with bistable dynamics: Cytoplasmic and genetic analogues of Allee effects. Am. Nat. 2011, 178, E48-E75. [CrossRef]

41. Schmidt, T.L.; Barton, N.H.; Rasic, G.; Turley, A.P.; Montgomery, B.L.; Iturbe-Ormaetxe, I.; Cook, P.E.; Ryan, P.A.; Ritchie, S.A.; Hoffmann, A.A.; et al. Local introduction and heterogeneous spatial spread of dengue-suppressing Wolbachia through an urban population of Aedes aegypti. PLoS Biol. 2017, 15, e2001894. [CrossRef]

42. Hoffmann, A.A.; Montgomery, B.L.; Popovici, J.; Iturbe-Ormaetxe, I.; Johnson, P.H.; Muzzi, F.; Greenfield, M.; Durkan, M.; Leong, Y.S.; Dong, Y.; et al. Successful establishment of Wolbachia in Aedes populations to suppress dengue transmission. Nature 2011, 476, 454-457. [CrossRef] [PubMed]

43. Schmidt, T.L.; Filipovic, I.; Hoffmann, A.A.; Rasic, G. Fine-scale landscape genomics helps explain the slow spatial spread of Wolbachia through the Aedes aegypti population in Cairns, Australia. Heredity 2018, 120, 386-395. [CrossRef] [PubMed]

44. Bakovic, V.; Stauffer, C.; Schuler, H. Allochronic isolation as a potential factor for prezygotic isolation in Rhagoletis cerasi (L.) (Diptera, Tephritidae). Mitt. Dtsch. Ges. Allg. Angew. Entomol. 2018, 21, 67-70.

45. Schuler, H.; Kern, P.; Arthofer, W.; Vogt, H.; Fischer, M.; Stauffer, C.; Riegler, M. Wolbachia in parasitoids attacking native European and introduced Eastern cherry fruit flies in Europe. Environ. Entomol. 2016, 45, 1424-1431. [CrossRef]

46. Heath, B.D.; Butcher, R.D.J.; Whitfield, W.G.F.; Hubbard, S.F. Horizontal transfer of Wolbachia between phylogenetically distant insect species by a naturally occurring mechanism. Curr. Biol. 1999, 9, 313-316. [CrossRef]

47. Huigens, M.E.; De Almeida, R.P.; Boons, P.A.H.; Luck, R.F.; Stouthamer, R. Natural interspecific and intraspecific horizontal transfer of parthenogenesis-inducing Wolbachia in Trichogramma wasps. Proc. R. Soc. Lond. Biol. 2004, 271, 509-515. [CrossRef]

48. Ahmed, M.Z.; Li, S.J.; Xue, X.; Yin, X.J.; Ren, S.X.; Jiggins, F.M.; Greeff, J.M.; Qiu, B.L. The intracellular bacterium Wolbachia uses parasitoid wasps as phoretic vectors for efficient horizontal transmission. PLoS Pathog. 2015, 11, 1004672. [CrossRef]

(C) 2019 by the authors. Licensee MDPI, Basel, Switzerland. This article is an open access article distributed under the terms and conditions of the Creative Commons Attribution (CC BY) license (http://creativecommons.org/licenses/by/4.0/). 